
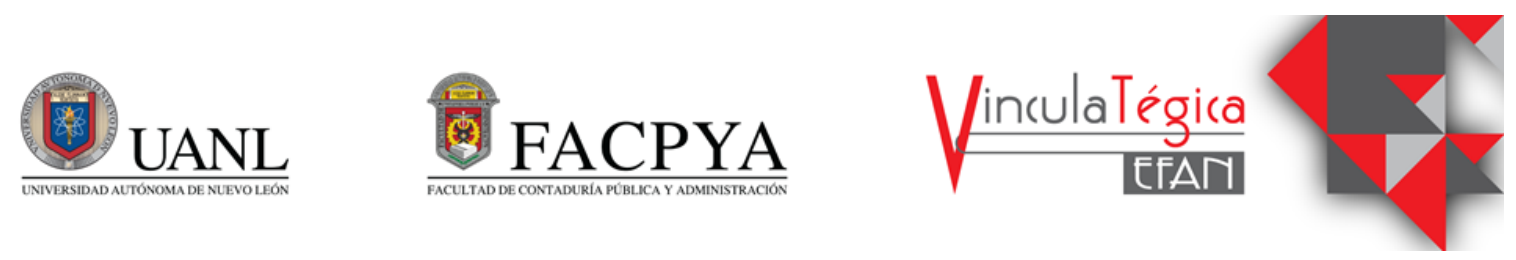

\title{
Modelación estructural de ecuaciones, metodología aplicada en una investigación: determinantes de la estructura de capital que afectan el valor de las empresas que cotizan en la BMV
}

\author{
Natalia Carolina Castillo Caballero ${ }^{1}$ y Juan Rositas Martínez ${ }^{2}$ \\ ${ }^{1}$ Universidad Autónoma de Nuevo León, Facultad de Contaduría Pública y Administración, \\ natalia.castillocb@uanl.edu.mx, Monterrey,Nuevo León, México. \\ ${ }^{2}$ Universidad Autónoma de Nuevo León, Facultad de Contaduría Pública y Administración \\ jrositasm@yahoo.com, Monterrey, Nuevo León, México.
}

\author{
Información del artículo revisado por pares \\ Fecha de aceptación: junio-2021 \\ Fecha de publicación en línea: diciembre-2021 \\ DOI: https://doi.org/10.29105/vtga7.2-13
}

\begin{abstract}
Resumen
Abstract

El objetivo de este artículo es presentar The objective of this article is to present information información que fomente el uso del método that encourages the use of the statistical method: estadístico: Modelación Estructural de Structural Modeling of Equations with a Partial Least Ecuaciones con enfoque de Mínimos Cuadrados Squares approach, in the area of Finance in Mexico, Parciales, en el área de Finanzas en México, ya se it can already be observed little used in this matter puede observar poco utilizado en esta materia compared to the usual use of the multiple regression, comparado con el uso habitual de la regresión having benefits that would allow to give another múltiple, teniendo bondades que permitirían darle approach to the investigations. This is intended to be otro enfoque a las investigaciones. Esto se achieved by means of a Theoretical Framework that pretende lograr por medio de un Marco Teórico explains the characteristics of this statistical method que explica las características de este método and proposing how an investigation is structured in estadístico y planteando cómo se estructura una this case. Then, to understand its application and the investigación en este caso. Después, para entender results that will be obtained, an illustrative example su aplicación y los resultados que se obtendrán se of conceptual-empirical application of this statistical presenta un ejemplo de aplicación conceptual - method is presented, applying it to the investigation empírica ilustrativa, de este método estadístico, of the Determinants of Capital Structure in companies aplicándolo a la investigación de los listed on the BMV, with a sample - test of 10 Determinantes de la Estructura de Capital en las companies listed on the BMV of the sector: Frequent empresas que cotizan en la BMV, con una muestra Consumer Products: Food and Beverage subsectors, - prueba de 10 empresas que cotizan en la BMV analyzing the annual data from 2010 to 2019. The del sector: Productos de Consumo Frecuente: main contribution is to present the main benefits of subsectores Alimentos y Bebidas, analizando los this statistical method, through the analysis of the datos anuales del 2010 al 2019. Como principal results obtained in the illustrative empirical example, aportación es presentar las principales bondades which are: to be able to handle constructs made up of de este método estadístico, por medio del análisis several variables to better analyze the hypotheses, to de los resultados obtenidos en el ejemplo empírico be able to analyze several equations that are presented ilustrativo, que son: poder manejar constructos at the same time, to be able to analyze indirect effects conformados por varias variables para analizar through mediating variables, and to perform the mejor las hipótesis, lograr analizar varias analysis of routes or dependencies, checking theories. ecuaciones que se presentan al mismo tiempo,
\end{abstract}


conseguir analizar efectos indirectos a través de variables mediadoras, y realizar el análisis de rutas o dependencias, comprobando teorías.

Palabras clave: Modelación Estructural de Ecuaciones, Mínimos Cuadrados Parciales, Determinantes, Estructura de Capital, Valor de la empresa.

JEL: G32, C50, C51.

\section{INTRODUCCIÓN}

En este artículo se presenta una forma metodológica que en que se puede llevar a cabo una investigación de Modelación Estructural de Ecuaciones, aplicándola en a la investigación Determinantes de la estructura de capital que afectan el valor de las empresas mexicanas que cotizan en la BMV, un análisis por sector.

Este artículo pretende presentar una forma de estadística muy innovadora e interesante que puede ser aplicable a muchas investigaciones en las ciencias sociales: $\mathrm{La}$ Modelación de Estructural de Ecuaciones.

El problema, en este caso, se presenta al desconocer esta técnica estadística relativamente nueva e innovadora (Jairo, 2008), especialmente si hablamos de su escaso uso en el área de Finanzas en México, en comparación con el uso habitual de la ecuación de regresión múltiple en la investigación, pudiéndose darle un foque más integral con la Modelación Estructural de Ecuaciones, por lo que se presenta como se puede utilizar en el proyecto: Determinantes de la Estructura de Capital que afectan el valor de las empresas que cotizan en la BMV: un análisis por sector.

La hipótesis, entonces, de este artículo sería que la manera de resolver un problema de investigación en donde se intenta explicar relaciones existentes, de manera simultánea, entre las variables que conforman la investigación, ya sean directas o indirectas, por medio de constructos formados por análisis factorial, es por medio la técnica estadística de Modelación Estructural de Ecuaciones. En este caso, en específico la técnica de mínimos cuadrados parciales (PLS
Key words: Structural Modeling of Equations, Partial Least Squares, Determinants, Capital Structure, Company Value.

JEL: G32, C50, C51.

por sus siglas en inglés).

Para resolver este problema, y contestar la hipótesis, se iniciará con un Marco Teórico que explica las características esenciales de la técnica estadística de Modelación Estructural de Ecuaciones, así como la técnica específica del PLS. Se presenta también en este apartado, investigaciones que explican por qué, bajo ciertas características, es mejor utilizar la técnica estadística de Modelación Estructural de Ecuaciones en las investigaciones acerca de los Determinantes de la Estructura de Capital.

Después se presenta la metodología que se siguió para hacer uso del método estadístico: Modelación de Ecuaciones Estructurales, en una muestra - prueba de los determinantes de la estructura de capital que afecta el valor de las empresas que cotizan en la BMV del Sector: Productos de Consumo Frecuente, subsectores: Alimentos y Bebidas. Se presenta los tipos de investigación, la forma de recolección de datos y los métodos estadísticos que se aplicaran.

Seguido a esto, se presentan los resultados obtenidos de la utilización del método Modelación Estructural de Ecuaciones en una muestra-prueba de los determinantes de la estructura de capital que afecta el valor de las empresas que cotizan en la BMV del Sector: Productos de Consumo Frecuente, subsectores: Alimentos y Bebidas. Esto es solo un ejemplo de aplicación conceptual empírica ilustrativa de este método estadístico. Los resultados finales de la investigación: Determinantes de la estructura de capital que afectan el valor de las empresas que cotizan en la BMV forman parte de un proyecto mayor.

Se termina con algunas conclusiones acerca de la utilización de este método estadístico para próximas investigaciones en el 
área de la Ciencias Sociales, y su bondad para comprobar hipótesis respaldadas por la teoría, con efectos directos, indirectos y constructos.

\section{MARCO TEÓRICO}

El Modelo Estructural de Ecuaciones es una novedosa técnica estadística multivariante que analiza varias relaciones de dependencia al mismo tiempo (Sergio Armando Guerra Moya \& Ponce Sánchez, 2014).

Estos modelos se pueden considerar una extensión de los modelos de Análisis de Regresión Múltiple, pero son mucho más flexibles y menos restrictivos. Se puede decir que el Modelo Estructural de Ecuaciones son varios modelos de análisis factorial que admiten efectos directos e indirectos entre los factores (Ruiz et al., 2010).

Este tipo de modelos son utilizado en las ciencias sociales, ya que estas estudian asiduamente conceptos abstractos, llamados también constructos, los cuales sólo se miden indirectamente a través de indicadores (Sergio Armando Guerra Moya \& Ponce Sánchez, 2014).

Como una gran ventaja de este tipo de modelos es que se permite proponer el tipo y dirección de relaciones, siendo un modelo de análisis de rutas para comprobar teorías, es decir, de analizar modelos causales.

\section{Modelo estructural}

El Modelo Estructural de Ecuaciones se conforman por dos modelos: el de medida y el de relaciones estructurales.

El modelo de medida es el de los indicadores observables, representados en rectángulos, que conforman los constructos, representados en círculos. Mientras que el modelo de relaciones estructurales es el que se está buscando comprobar a través de la teoría, donde se presentan los efectos y relaciones entre los constructos, siendo estos generalmente variables latentes.

\section{Clases de variables}

De acuerdo con su cometido y su medición las variables de un modelo estructural pueden ser:

- Indicador o variable observable. Son las variables medibles.
- Variable latente. Característica no observable que se desea medir.

- Variable error. Son los errores inherentes a las variables, además de lo que no fue contemplado para medir en el modelo.

- Variable por grupo. Representa subpoblaciones.

- Variable exógena. Variable que afecta, pero no es afectada.

- Variable endógena. Variable que recibe un efecto.

\section{Representación de un modelo estructural.}

Para establecer un modelo estructural de efectos, causas y relaciones, regularmente se crea un diagrama causal o diagrama de rutas, las cuales se representan de la siguiente manera: Los indicadores $\mathrm{o}$ variables observables se representan por medio de rectángulos. Las variables latentes se simbolizan a través de círculos. Los errores se plasman libres. Mientras que los efectos o relaciones se representan por medio de flechas, es importante aclarar que los parámetros del modelo se incluyen sobre las flechas.

\section{Relación Causal}

En la técnica de Modelo Estructural de Ecuaciones se pueden distinguir diversos tipos de relaciones.

Es importante señalar que la mera asociación entre variables no crea la causalidad, la condición suficiente para que esta exista es que: "Una variable A es causada de $\mathrm{B}$ si siempre que se da A acontece $\mathrm{B}$, $\mathrm{y}$ nunca acontece B si previamente no se ha dado A" (Sergio Armando Guerra Moya \& Ponce Sánchez, 2014).

\section{Tipos de relación causal}

Los modelos causales hacen referencia a un "análisis de trayectoria" que es el estudio de las variables que causan y las que tienen el efecto.

La variable efecto se denomina variable dependiente, endógena o explicada, y la variable que causan la anterior son nombradas, independientes, exógenas o explicadas. 
El análisis de trayectoria es un método estadístico parecido a la regresión, pero con capacidad explicativa, estudiante los efectos directos e indirectos en las variables observables.

Los tipos de relación causal son: los directos, indirectos, y espurios.

La relación directa es cuando una variable causa la otra.

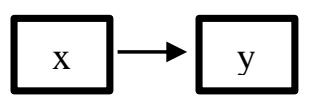

La relación indirecta existe cuando se presentan tres variables, existiendo una mediadora.

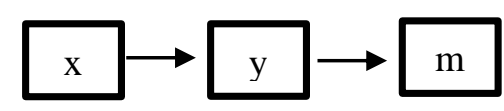

La relación espuria es cuando prevalece cuando hay covariación entre dos variables, pero no implica necesariamente la relación causal, esta puede ser ficticia. Por ejemplo, la estatura de un niño con la inteligencia, la estatura de un niño puede asociarse perfectamente la inteligencia, pero esto no quiere decir que la cause.

Los efectos totales nos hablan de la suma de los efectos directos, indirectos y los efectos no analizados.

\section{Técnica de Mínimos Cuadrados Parciales}

En el Modelo Estructural de Ecuaciones con enfoque de covarianza se tiene la rigidez del uso de la covarianza para estimar sus parámetros, para lo cual se tiene que cumplir con aspectos como la normalidad de los datos, así como la necesidad de una gran cantidad de datos para su análisis (Layva Cordero \& Olague, 2014)

Debido a la inflexibilidad anteriormente expuesta se creó la Técnica de Mínimos Cuadrados Parciales, como una excelente alternativa de análisis.

En el caso de la regresión con PLS se trata de identificar los elementos sustanciales de la variable independiente, que además sean convenientes para explicar la variable dependiente. Para eso, la regresión PLS construye variables latentes con la mayor variación de las variables independientes observadas, modelando al mismo tiempo la variable dependiente.

Este tipo de análisis es de especial ventaja para el análisis causal predictivo, cuando el problema a resolver es muy complejo, con poca teoría de respaldo.

Es preciso mencionar que, de acuerdo con Rositas (2007) "los softwares para el MES se categorizan según el enfoque o enfoque de covarianza (Lisrel, EQS, AMOS, por ejemplo) o enfoques de Mínimos Cuadrados Parciales (PLSGraph, Smart PLS, etc.)"

\section{Algunos de los criterios para elegir Análisis PLS en el desarrollo de un problema de investigación (Layva Cordero \& Olague, 2014).}

- $\quad$ Si se tiene como objetivo determinar constructos, explicativos o explicados.

- En caso de que la investigación sea exploratoria, o una extensión de la teoría existente.

- Si el modelo estructural es complejo.

- En el caso de que el tamaño de la muestra sea pequeño.

- Si los datos son anormales.

Criterios por la que usar PLS-SEM en lugar de una regresión en el análisis de los Determinantes de la Estructura de Capital. De acuerdo con Ramli (2018), se debe utilizar PLS-SEM (Partial Least Structure - Structure Ecuations Model por sus siglas en inglés), en estudios de los Determinantes de la Estructura de Capital cuando en la investigación:

- Existe una variable mediadora. Es decir, se espera un efecto a través de una variable para llegar a otra variable.

- Cuando existan relaciones simultáneas en el estudio.

- En el caso de que los datos compongan un constructo latente, en un análisis factorial.

- Cuando se quiera evaluar modelos de análisis de rutas o dependencias, y así comprobar teorías. 
Es importante mencionar que para el ejemplo de aplicación conceptual - empírico ilustrativo del método estadístico: Modelación Estructural de Ecuaciones, con el tema Determinantes de la Estructura de Capital que afectan el valor de la empresas que cotizan en la BMV, análisis del Sector Productos de consumo frecuentes, subsectores: alimentos y bebidas, se tienen como variables independientes: Desarrollo del mercado de capitales, tipo de cambio, riesgo de mercado, liquidez, riesgo de negocio, oportunidad de crecimiento, estructura fiscal, estructura del activo y tamaño de la firma, y como variables dependientes, la estructura de capital, y el valor de la firma, donde esta última depende de la estructura de capital (variable mediadora) y de las variables independientes. Estas variables están fundamentadas teóricamente en un proyecto más grande, sin embargo, el único objetivo de este artículo es presentar la metodología.

\section{MÉTODO}

A continuación, se plantea la metodología que se seguirá para llevar a cabo el ejemplo de aplicación conceptual - empírica ilustrativa de este método estadístico: Modelación Estructural de Ecuaciones, en la muestraprueba del SECTOR PRODUCTOS DE CONSUMO FRECUENTE, subsectores: Alimentos y Bebidas.

\section{Tipo y diseño de la investigación.}

Los tipos de investigación que se llevarán a cabo son:

- Exploratoria: $\mathrm{Se}$ recolecta información relevante para conformar el Modelo de Ecuaciones Estructurales.

- Descriptiva: Se presentan los datos descriptivos de las variables independientes y dependientes de la modelación estructural de ecuaciones.

- Correlacional: es necesario presentar la correlación existente entre las variables dependientes "estructura de capital" y "valor de la empresa" con las variables independientes que pudieran estar influenciando en ellas.

\section{Diseño de la investigación}

Este estudio es de tipo no experimental, ya que las variables de la investigación no se van a manipular, se observarán en su contexto natural.

Además, es longitudinal y transeccional, pues en esta muestra - prueba, se van a analizar a diferentes empresas del sector Productos de Consumo Frecuente: subsectores Alimentos y Bebidas de las empresas que cotizan en la BMV, en un periodo de tiempo de diez años, del 2009 al 2019. Los datos son anuales.

Para determinar si las variables independientes tienen influencia en la variable dependiente "estructura de capital" y "valor de la empresa" se utilizará el estadístico " $t$ Student" con un nivel de significancia del 10\% y $5 \%$.

De la misma forma se analizará la "Rcuadrada ajustada" para observar el porcentaje de variación de la variable dependiente que se explica colectivamente por todas las variables independientes.

\section{Método de recolección de datos}

Los datos de tipo secundario, fueron obtenidos de la base de datos "Bloomberg", la que es una empresa de información financiera y de noticias mundial (Bloomberg, 2020), este ente ofrece información confiable de las empresas, donde se obtuvo la información de las empresas que cotizan en la BMV, así como datos económicos y financieros, como el riesgo de mercado en el lapso de tiempo estudiado. De examinaron páginas oficiales de la Bolsa Mexicana de Valores, del Banco de México, INEGI y Yahoo Finanzas para poder obtener datos relevantes de la información. Consiguiendo, con esta recolección datos reales y confiables, de las variables dependientes e independientes propuestas en el marco teórico.

\section{Métodos estadísticos utilizados}

A continuación, se presentan los métodos estadísticos que se consideran más apropiados para el análisis de los datos recolectados.

Se iniciará con el correspondiente análisis descriptivo, el correlacional 
de multicolinealidad y por último el análisis de Ecuaciones Estructurales. Estos pasos se muestran en la Ilustración 1. estos casos se pueden formular y probar hipótesis más exactas (Martínez \& Sepúlveda, 2012). Los dos análisis factoriales

Ilustración 1. Pasos del análisis de los datos recolectados.

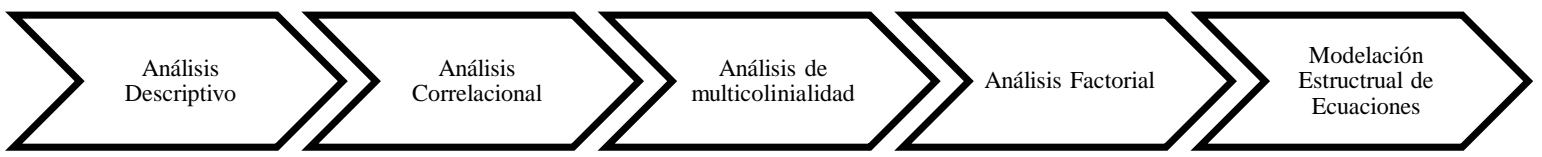

Elaboración propia basado en (Ríos Ángeles, 2019)

La primera etapa es el análisis descriptivo donde se presentan las medidas de tendencia central (media, mediana y moda) y dispersión (desviación estándar y varianza).

Después se observará la correlación de las variables, observándose la magnitud de relación existente entre las variables de estudio. De acuerdo con Restrepo (2007) tanto el método de correlación de Pearson como Spearman son coeficientes de correlación son técnicas bivariados que se empelan en el campo multivariado. El coeficiente de correlación mide el grado de asociación que existe entre dos variables aleatorias este fluctúa entre $-1 \leq \rho \leq 1$.

Además, se realizará el análisis de multicolinealidad de las variables. La multicolinealidad describe la dependencia lineal existente entre las variables predictoras(Vega Vilca \& Guzman, 2011). Aunque es un problema que se dificulta medir con exactitud, regularmente se calcula con el Factor de Inflación de Varianza (VIF, por sus siglas en inglés). Generalmente se considera que existe multicolinealidad cuando VIF $\geq 10$.

El siguiente paso es realizar el análisis factorial, existe el exploratorio y el confirmatorio, de acuerdo con Martínez \& Sepúlveda (2012). En el caso del exploratorio es cuando se intenta definir grupos de variables que estén altamente correlacionados entre sí. Este tipo de análisis también se usa para reducir la complejidad de un análisis con muchas variables, reduciendo su número. En el caso del análisis exploratorio no se necesita conocimiento a priori para estructurar los factores. Este análisis también se usa para eliminar variables poco relevantes o muy colineales. Por otro lado, el análisis factorial confirmatorio se evalúa un conjunto de factores que están respaldados por la teoría. En anteriormente explicados no son excluyentes.

En el caso de este estudio el análisis factorial que se hará será el confirmatorio, puesto que los constructos que se proponen tienen la teoría por respaldo. Es necesario indicar que, en el programa que se realizará el Modelo Estructural de Ecuaciones de Mínimos Cuadrados Ordinarios llamado Smart-PLS3, realiza, al mismo tiempo del análisis estructural, el análisis factorial de los constructos planteados, presentando el coeficiente de cada variable que conforma el constructo.

Como siguiente paso se realizará el análisis de inferencias. Lo que se intenta en esta investigación es conocer la relación existente entre las variables independientes "determinantes de la estructura de capital" y las variables dependientes "estructura de capital", y "valor de la empresa", se necesita realizar el análisis multivariable, pues de manera simultánea se intenta medir, explicar y predecir las relaciones existentes, entre los elementos que se analizan en la investigación. Los datos obtenidos deben ser escrupulosamente interpretados por el investigador (Sergio A. Guerra Moya, 2007).

Es preciso mencionar que en la aplicación conceptual - empírica ilustrativa propuesta de los "Determinantes de la Estructura de Capital que afectan el valor de las empresas que cotizan en la BMV, un análisis por sector", se pretenden observar las relaciones causales entre las variables sujetas de estudio, y al mismo tiempo se necesita analizar varias relaciones de dependencia que se presentan simultáneamente la mejor forma de analizar los datos es con el método estadístico de Modelación Estructural de Ecuaciones (Sergio Armando Guerra Moya \& Ponce Sánchez, 2014). 
La Modelación Estructural de Ecuaciones es una extensión del Análisis de Regresión Múltiple, en este caso se puede analizar la estructura subyacente de cada variable, donde, además, estas pueden actuar como variables independientes de las diferentes ecuaciones del modelo. La existencia de dependencia de estos modelos ocurren en varios niveles(Sergio Armando Guerra Moya \& Ponce Sánchez, 2014).

En esta investigación que plantea el Modelo Estructural de Ecuaciones para una prueba piloto de los determinantes de la estructura de capital que afecta el valor de las empresas que cotizan en la BMV del Sector: Productos de Consumo Frecuente, subsectores: Alimentos y Bebidas, el modelo sería el que se presenta en la Ilustración 2, donde se presenta el modelo de medida (rectángulos) y el modelo estructural (círculos). Es preciso mencionar que los círculos son constructos creados por los indicadores observables, expuestos como rectángulos.

En la ilustración 2 además se observa las relaciones existentes entre los constructos, y de las variables observables o indicadores con los constructos, como modelo de análisis de ruta. Las cuales representan la hipótesis formulada en la investigación original, los determinantes de la estructura de capital que afectan el valor de las empresas, directa o indirectamente, que cotizan en la BMV: un análisis por sector, son, el desarrollo del mercado de capitales, el tipo de cambio, el riesgo de mercado, la liquidez, el riesgo de negocio, la oportunidad de crecimiento, el escudo fiscal, la estructura de los activos y el tamaño de la firma.

Las ecuaciones de los efectos directos serían:
Estructura de capital

$=\beta_{1}$ desarrollo del mercado de capitales

$+\beta_{2}$ tipo de cambio

$+\beta_{3}$ el riesgo de mercado $+\beta_{4}$ liquidez

$+\beta_{5}$ riesgo de negocio

$+\beta_{6}$ oportunidad de crecimiento

$+\beta_{7}$ escudo fiscal

$+\beta_{8}$ estructura de los activos

$+\beta_{9}$ tamaño de la firma $+\varepsilon$

Valor de la empresa $=$

$\beta_{1}$ estructura de capital $+\varepsilon$

Valor de la empresa

$=\beta_{1}$ desarrollo del mercado de capitales

$+\beta_{2}$ tipo de cambio

$+\beta_{3}$ el riesgo de mercado $+\beta_{4}$ liquidez

$+\beta_{5}$ riesgo de negocio

$+\beta_{6}$ oportunidad de crecimiento

$+\beta_{7}$ escudo fiscal

$+\beta_{8}$ estructura de los activos

$+\beta_{9}$ tamaño de la firma $+\varepsilon$

$$
y_{n}=f\left(x_{n 1}, x_{n 2}, \ldots, x_{n m}\right)
$$

En este caso los constructos o variables latentes serían: la estructura de capital, el valor de la empresa y cada uno de los determinantes 
Ilustración 2 Diagrama de modelo estructural y de medible (con su forma de cálculo)

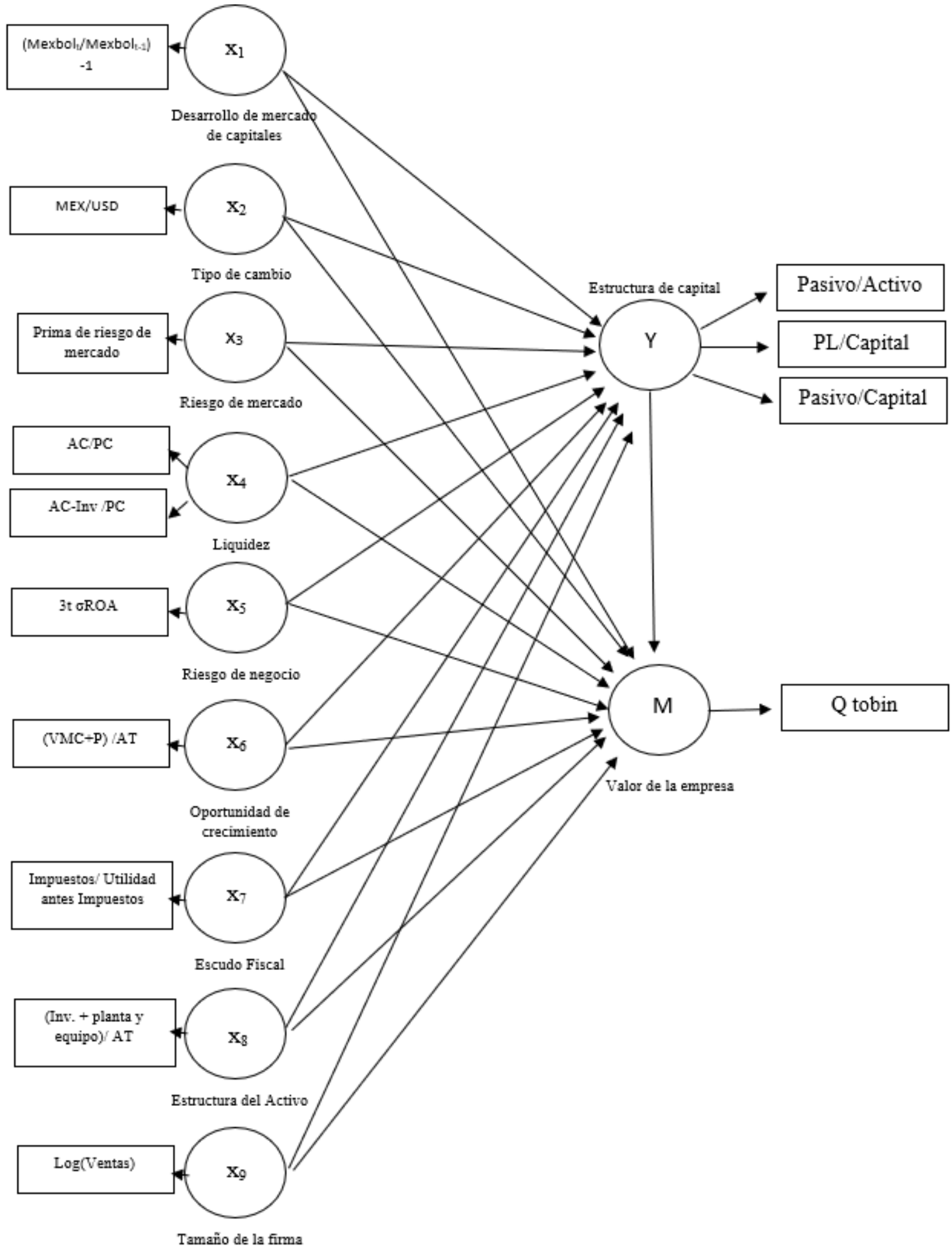

Fuente: Elaboración propia, las fórmulas de cada indicador observable se obtuvieron de diversos autores, fundamentados teóricamente. 
de la estructura de capital, y las variables observables o indicadores serían los resultados de las ecuaciones hechas con datos obtenidos a partir de sistemas de información financiera como Bloomberg, Banxico, Yahoo Finanzas. Estas ecuaciones fueron fundamentadas teóricamente.

De acuerdo con Flores del Ángel (2021) dentro del análisis de la Modelación Estructural de Ecuaciones se pueden observar tres elementos: a) el modelo de medición b) los coeficientes de trayectoria (path coefficients) para la relación del modelo estructural; y c) y los resultados obtenidos de la variable mediadora.

Para valuar el modelo de medición existen varios pasos, el primero es calcular el coeficiente del Alfa de Cronbach. Este coeficiente es un indicador de confiablidad del cálculo de un constructo o una dimensión, el valor de este oscila entre 0.00 y 1.00 (Rositas Martínez, 2021) Para que el cálculo del constructo se considere aceptable, el Alfa debe ser mínimo de 0.60. Después se debe valorar la consistencia de cada indicador, para esto se analiza las cargas externas (outer loadings), estos valores deben ser mayores a 0.70. El siguiente paso es observar la validez convergente, que se mide por medio de la varianza promedio extradía (AVE), su valor mínimo es 0.50 para ser aceptable (Flores del Ángel, 2021).
Una vez obtenidos resultados positivos en todos los análisis hechos al modelo de medición, entonces se puede valuar el Modelo Estructural. Se inicia observado la multicolinealidad a través del Factor de Inflación de la Varianza (VIF), el cual debe ser menor a 5.0. Después se analizan los coeficientes de las relaciones propuestas en la hipótesis, que serían los coeficientes trayectoria, así como su significancia, utilizando la técnica Bootstrapping. El siguiente paso es observar el coeficiente de determinación $\left(\mathrm{R}^{2}\right)$, con lo que se valora el grado de explicación del modelo estructural, pueden existir varios coeficientes de determinación de acuerdo con el análisis que se realiza.

El último elemento para analizar en una Modelo Estructural de Ecuaciones es el de los efectos indirectos de las variables, los cuales también se obtienen con la técnica Bootstrapping.

Una vez analizado estos tres elementos del Modelo Estructural de Ecuaciones se debe proceder a la comprobación de la hipótesis.

\section{RESULTADOS}

A continuación, se presentan los resultados del análisis hecho con el método estadístico Modelación Estructural de Ecuaciones, a los datos secundarios obtenidos, como muestra -prueba, en páginas

Tabla 1 Estadísticos Descriptivos. Subsectores: Alimentos y Bebidas. Empresas que cotizan en la BMV 2010-2019

\begin{tabular}{|c|c|c|c|c|c|c|c|c|c|}
\hline & $\mathrm{N}$ & Rango & Minimo & Máximo & Media & Desv. tip. & Varianza & Asimetria & Curtosis \\
\hline & Estadistico & Estadistico & Estadistico & Estadistico & Estadistico & Estadistico & Estadistico & Estadistico & Estadistico \\
\hline $\begin{array}{l}\text { Desarrollo_Mercado_C } \\
\text { apitales }\end{array}$ & 100 & .36 & -.16 & .20 & .0357 & .09967 & .010 & -.027 & -.310 \\
\hline Liquidez & 100 & 3.76 & .64 & 4.40 & 1.7984 & .88393 & .781 & 1.079 & .374 \\
\hline Prueba_de_acido & 100 & 2.80 & .40 & 3.19 & 1.2661 & .63597 & .404 & 1.378 & 1.159 \\
\hline Riesgo_de_negocio & 100 & .25 & .00 & .25 & .0309 & .04551 & .002 & 3.763 & 15.356 \\
\hline $\begin{array}{l}\text { Oportunidad_de_crecim } \\
\text { iento }\end{array}$ & 100 & 2.28 & .66 & 2.94 & 1.5265 & .44695 & .200 & .692 & .822 \\
\hline Escudo_Fiscal & 100 & 5.68 & -4.68 & 1.00 & .2055 & .54143 & .293 & -7.694 & 68.589 \\
\hline Apalancamiento & 100 & .59 & .14 & .72 & .4352 & .13635 & .019 & .282 & -.284 \\
\hline Endevdamiento_1 & 100 & 1.85 & .02 & 1.87 & .5255 & .44004 & .194 & 1.406 & 1.599 \\
\hline Endevdamiento_2 & 100 & 2.47 & .16 & 2.63 & .9012 & .57574 & .331 & 1.443 & 1.483 \\
\hline Valor_de_la_emrpesa & 100 & 2.31 & .66 & 2.98 & 1.6172 & .43257 & .187 & .496 & .887 \\
\hline Riesgo_de_mercado & 100 & .04 & .04 & .08 & .0531 & .01333 & .000 & .596 & -.726 \\
\hline Tipo_de_cambio & 100 & 8.27 & 12.35 & 20.62 & 16.3135 & 3.07641 & 9.464 & .076 & -1.709 \\
\hline Tamaño_de_la_empres & 100 & 2.09 & 3.62 & 5.70 & 4.6244 & .60807 & .370 & -.121 & -1.200 \\
\hline Estructura_del_activo & 100 & .59 & .17 & .76 & .4275 & .14877 & .022 & .379 & -1.089 \\
\hline $\mathrm{N}$ válido (según lista) & 100 & & & & & & & & \\
\hline
\end{tabular}


de finanzas como Bloomberg o Banxico, del sector: Productos de Consumo frecuente; subsectores: Alimentos y Bebidas, de 10 empresas que cotizan en la BMV, años 2010 al 2019, con datos anuales.

Se inicia con el análisis estadístico descriptivo de las variables, las cuales se presentan en la tabla 1 .

En estos estadísticos descriptivos se puede apreciar que en la ratio de apalancamiento se tiene una media de .4352 , esto quiere decir que, en estos subsectores, en promedio, $43 \%$ de sus activos están financiados con deuda. También se puede observar que el Máximo de apalancamiento es de .72, y el mínimo .14.

Se realizaron así mismo la tabla de correlaciones, y se observó que algunas variables tienen una buena correlación con las variables dependientes.

Para analizar el modelo de medición, para iniciar se analizó el alfa de Cronbach, obteniendo los resultados que se observan en la tabla 2. De acuerdo con los números obtenidos, se observa que los constructos sugeridos por la teoría son confiables

\section{Tabla 2 Alfa de Cronbach}

\begin{tabular}{lr}
\hline & Alfa de Cronbach \\
\hline Desarrollo del mercado de capitales & 1.000 \\
Escudo Fiscal & 1.000 \\
Estructura de capital & 0.978 \\
Estructura del Activo & 1.000 \\
Liquidez & 0.973 \\
Oportunidades de Crecimiento & 1.000 \\
Riesgo de mercado & 1.000 \\
Riesgo de negocio & 1.000 \\
Tamaño de la firma & 1.000 \\
Tipo de Cambio & 1.000 \\
Valor de la empresa & 1.000 \\
\hline
\end{tabular}

Fuente: Elaboración propia con datos obtenidos de bases de datos como Bloomberg y Banxico.

Después se realizó las pruebas de cargas externas del modelo y la validez convergente del modelo de medición, obteniendo buenos resultados.

$\mathrm{Al}$ realizar este proceso, se procedió a evaluar el modelo estructural, iniciando con la prueba de multicolinealidad de las variables, obteniendo los resultados de la tabla 3. Aquí se puede observar el Factor de Inflación de la Varianza obteniendo en la mayoría de las variables independientes e independientes, en el modelo estructural como indicadores, la mayoría tienen un VIF menor de 5.0. Se puede observar que las variables que obtuvieron un VIF mayor que 5.0 son las variables que conforman los constructos llamados: Estructura de Capital conformados por Apalancamiento, Endeudamiento 1 y Endeudamiento 2, y las variables de constructo liquidez, conformado por liquidez y Prueba de ácido.

Tabla 3 Factor de Inflación de la Varianza

\begin{tabular}{lr}
\hline & VIF \\
\hline Apalancamiento & 10.499 \\
Desarrollo mercado de capitales & 1.000 \\
Endeudamiento 1 & 12.153 \\
Endeudamiento 2 & 23.554 \\
Escudo Fiscal & 1.000 \\
Estructura del activo & 1.000 \\
Liquidez & 9.891 \\
Oportunidad de crecimiento & 1.000 \\
Prueba de ácido & 9.891 \\
Riesgo de mercado & 1.000 \\
Riesgo de negocio & 1.000 \\
Tamaño de la empresa & 1.000 \\
Tipo de cambio & 1.000 \\
Valor de la empresa & 1.000 \\
\hline
\end{tabular}

Fuente: Elaboración propia con datos obtenidos de bases de datos como Bloomberg y Banxico.

Para proseguir con el análisis del modelo estructural, se obtuvieron los coeficientes de cada una de las relaciones de los constructos independientes con las dependientes. Obteniendo los resultados de la tabla 4. 
Tabla 4 Coeficientes tipificados obtenidos en el Modelo Estructural para cada variable independiente que afecta directamente a una dependiente.

\begin{tabular}{lrr}
\hline & $\begin{array}{c}\text { Estructura de } \\
\text { capital }\end{array}$ & $\begin{array}{l}\text { Valor de la } \\
\text { empresa }\end{array}$ \\
\hline Desarrollo del mercado de & 0.015 & 0.015 \\
capitales & 0.121 & -0.022 \\
Escudo Fiscal & & -0.053 \\
Estructura de capital & 0.239 & -0.079 \\
Estructura del Activo & -0.404 & -0.038 \\
Liquidez & -0.053 & 0.951 \\
Oportunidad de crecimiento & 0.009 & -0.007 \\
Riesgo de mercado & -0.064 & -0.073 \\
Riesgo de negocio & 0.426 & -0.016 \\
Tamaño de firma & 0.049 & -0.010 \\
Tipo de cambio & & \\
Valor de la empresa & & \\
\hline
\end{tabular}

Fuente: Elaboración propia.

De la misma forma, se obtuvieron los pvalor de los coeficientes para poder saber la significancia de estos al 10\%, obteniendo los resultados de la tabla 5 .

Tabla 5 P-Valor de los coeficientes de la influencia de las variables independientes a las dependientes del Modelo Estructural - Hipótesis propuestas. SIGNIFICANCIA $10 \%$.

\begin{tabular}{|c|c|}
\hline & P-Valor \\
\hline Desarrollo del mercado de capitales $>$ Estructura de capital & 0.879 \\
\hline Desarrollo del mercado de capitales $>$ Valor de la empresa & 0.610 \\
\hline Escudo fiscal $>$ Estructura de capital & $0.084^{*}$ \\
\hline Escudo fiscal $>$ Valor de la emrpesa & 0.574 \\
\hline Estructura de capital $>$ Valor de la empresa & 0.150 \\
\hline Estructura del Activo $>$ Estructura de capital & $0.020 * *$ \\
\hline Estructura del Activo $>$ Valor de la empresa & $0.042 * *$ \\
\hline Liquidez $>$ Estructura de capital & $0.000 * * *$ \\
\hline Liquidez $>$ Valor de la empresa & 0.270 \\
\hline Oportunidades de crecimiento $>$ Estructura de capital & 0.502 \\
\hline Oportunidades de crecimiento $>$ Valor de la empresa & $0.000 * * *$ \\
\hline Riesgo de mercado $>$ Estructura de capital & 0.913 \\
\hline Riesgo de mercado $\rightarrow$ Valor de la empresa & 0.800 \\
\hline Riesgo de negocio $>$ Estructura de capital & 0.566 \\
\hline Riesgo de negocio $\rightarrow$ Valor de la empresa & 0.137 \\
\hline Tamaño de la firma $\rightarrow$ Estructura de capital & $0.000 * * *$ \\
\hline Tamaño de la firma $>>$ Valor de la empresa & 0.669 \\
\hline Tipo de cambio $\rightarrow$ Estructura de capital & 0.595 \\
\hline Tipo de cambio $>$ Valor de la empresa & 0.754 \\
\hline
\end{tabular}

Fuentes: Elaboración propia con el programa SmartPLS3. Valores ***p $<0.01, * * \mathrm{p}<0.05, * \mathrm{p}<0.10$

En la Tabla 5 se puede observar que: el tamaño de la firma, la liquidez, la estructura del activo y el escudo fiscal están afectando la estructura de capital. Mientras que las variables que afectan el Valor de la empresa son: las oportunidades de crecimiento y la estructura del activo. Por lo que podemos observar que los determinantes propuestos podrían estar afectando la estructura de capital y al mismo tiempo, directamente, el valor de la empresa, estadísticamente hablando, con una significancia del $10 \%$. Esto en el sector: productos de consumo frecuente, subsectores: Alimentos y Bebidas, de las empresas que cotizan en la BMV.

De la misma forma, se obtuvieron los Coeficientes de Determinación $\mathrm{R}^{2}$ de las ecuaciones propuestas del modelo estructural. Los resultados se muestran en la tabla 6 .

Tabla 6 Coeficientes de Determinación de ambos modelos de dependencia propuestos

Coeficiente de Determinación $R^{2}$ de ambas variables dependientes

\begin{tabular}{lrr}
\hline & R cuadrada & $\begin{array}{c}\text { R cuadrada } \\
\text { ajustada }\end{array}$ \\
\hline Estructura de capital & 0.432 & 0.375 \\
Valor de la empresa & 0.950 & 0.944 \\
\hline
\end{tabular}

Fuente: Elaboración propia, analizado con datos tomados de fuentes como Bloomberg, Banxico, etc.

Se observaron, además, los efectos indirectos de las variables propuesta, con la técnica Bootstrapping. Los resultados aparecen en la Tabla 7. Sin embargo, ninguna relación indirecta fue significativa.

Tabla 7. Efectos indirectos de las variables a través de la variable mediadora - Significancia al $10 \%$

\begin{tabular}{lr}
\hline & P-Valor \\
\hline Escudo fiscal $>$ Estructura de capital $>$ Valor de la empresa & 0.440 \\
Liquidez $>$ Estructura de capital $>$ Valor de la empresa & 0.198 \\
& 0.937 \\
Riesgo de mercado $>$ Estructura de captial $>>$ Valor de la empresa & \\
& 0.255 \\
Estructura del activo $>$ Estructura de capital $>$ Valor de la empresa & \\
$\begin{array}{l}\text { Desarrollo del mercado de capitales }>\text { Estructura de capital }>\text { Valor } \\
\text { de la empresa }\end{array}$ & 0.910 \\
$\begin{array}{l}\text { Oportunidades de crecimiento }>\text { Estructura de capital }>\text { Valor de la } \\
\text { empresa }\end{array}$ & 0.698 \\
$\begin{array}{l}\text { Riesgo de negocio }>\text { Estructura de capital }>\text { Valor de la empresa } \\
\text { Tipo de cambio }>\text { Estructura de capital }>\text { Valor de la empresa }\end{array}$ & 0.698 \\
& 0.731 \\
Tamaño de la firma $>$ Estructura de capital $>$ Valor de la empresa & 0.162 \\
\hline
\end{tabular}

Fuente: Elaboración propia con el programa Smart-PLS3. 


\section{CONCLUSIONES}

En este ejemplo de aplicación conceptual empírica ilustrativa se pudo observar en los resultados que, en el análisis de los datos con la Modelación Estructural de Ecuaciones con enfoque de Mínimos Cuadrados Ordinarios, se puede utilizar constructos conformados por varias variables para la aplicación de las hipótesis que lo necesitan, analizando su confiabilidad por medio del Alfa de Cronbach, además este método factoriza los indicadores propuestos en el constructo.

Así mismo en los resultados, en el modelo estructural, se observa que se analiza el Factor de Inflación de la Varianza, para determinar el buen uso de las variables en las ecuaciones. Además, se estudian varias ecuaciones al mismo tiempo, como los efectos directos que tienen las variables independientes con las variables dependientes "estructura de capital" y el "valor de la empresa".

En los resultados también se observa el efecto que tiene la variable dependiente "estructura de capital", que se presenta como independiente, sobre la variable dependiente "valor de la empresa", analizando así su efecto directo, y los indirectos que tienen las variables independientes sobre la dependiente "valor de la empresa" a través de la variable mediadora "estructura de capital".

Como conclusiones podemos decir que para un estudio de los Determinantes de la Estructura es mejor realizar un análisis de Modelación Estructural de Ecuaciones, específicamente el método de Mínimos Cuadrados Parciales, cuando el estudio:

- Maneje constructos para analizar mejor las hipótesis.

- Existen varias ecuaciones que se analizan al mismo tiempo.

- Cuando existan variables mediadoras.

- Cuando existan análisis de rutas o dependencias, para con ello comprobar teorías.

Es preciso mencionar, además, de acuerdo con Layva \& Cordero (2014), se pueden observar las características tan bondadosas del método de Modelación Estructural de Ecuaciones, en específico el método Mínimos Cuadrados Parciales (PLS-SEM por sus siglas en ingles), como lo son:

- Se puede manejar si la investigación es exploratoria, o se quiere crear una extensión de alguna teoría ya existente.

- Cuando se tenga un tamaño de muestra pequeño.

- No hay necesidad de que los datos tengan distribución normal.

- Cuando el modelo a analizar sea complejo.

En este artículo se presenta, tanto en la teoría, como en la metodología y los resultados, una forma simple de cómo se lleva a cabo una investigación con el método de Modelación Estructural de Ecuaciones por Mínimos Cuadrados Parciales. Esto para ofrecer a los investigadores otra forma en que se pueden llevar a cabo muchos estudios en el área de las Ciencias Sociales, como en los estudios en Finanzas, y lograr un análisis más completo.

De hecho, es importante precisar que muchos autores han recomendado el uso de este método estadístico, Modelación Estructural de Ecuaciones con enfoque de Mínimos Cuadrados Parciales, para análisis de datos, con un enfoque integral, en muchas otras áreas de investigación por sus muchas bondades, como Rositas (2004) en su trabajo pionero "La gestión bajo una cultura de calidad en México: una verificación empírica.", donde aplica este método estadístico, y concluye animando al uso de este método estadístico con el enfoque de Mínimos Cuadrados Parciales (PLS por sus siglas en inglés), pues entre 
sus muchas ventajas está el que su uso implica menos restricciones como la de no exigencia de normalidad, tampoco precisa grande tamaños de muestra, siendo además más tolerante con la multicolinealidad.

Dado lo anterior, este artículo intenta fomentar el uso de la Modelación Estructural de Ecuaciones por medio de Mínimos Cuadrados Parciales, en el área de Finanzas en México.
Se puede decir que se logró el objetivo de este artículo que es presentar la Modelación Estructural de Ecuaciones como una excelente técnica de uso en la investigación para resolver problemas con relaciones causales de manera simultánea, por medio de constructos conformados con el análisis factorial.

\section{REFERENCIAS}

Bloomberg. (2020). https://www.bloomberg.com/latam/blog/category/mexico/

Flores del Ángel, P. (2021). Elementos de la Compensación variable que motivan a los trabajadores para incrementar su desempeño laboral basado en competencias, en las empresas japonesas del estado de Nuevo León: un modelo de ecuaciones estructurales.

Guerra Moya, Sergio A. (2007). Introducción al Análisis Multivariable (p. 24). Apuntes / Memoria en extenso.

Guerra Moya, Sergio Armando, \& Ponce Sánchez, R. (2014). Capítulo 15 Análsis Multivariante: Modelización con Ecuaciones Estructurales. In Métodos y técnicas cualitativas y cuantitativas aplicables a la investigación en ciencias sociales (pp. 315-350).

Jairo, I. (2008). The use of structural equation modelling (SEM) in capital structure empirical analysis. KCA Journal of Business Management, $1,1$.

Layva Cordero, O., \& Olague, J. T. (2014). Capítulo 22 Modelos de Ecuaciones Estructurales por el Método de Mínimos Cuadrados Parciales (Partial Least Squares-PLS). In Métodos y técnicas cualitativas y cuantitativas aplicables a la investigación en ciencias sociales (pp. 479-497).

Martínez, C. M., \& Sepúlveda, M. A. R. (2012). Introducción al análisis factorial exploratorio. Revista Colombiana de Psiquiatría, 41(1), 197-207. https://doi.org/10.1016/s00347450(14)60077-9

Ramli, N. A., Latan, H., \& Nartea, G. V. (2018). Why should PLS-SEM be used rather than regression? evidence from the capital structure perspective. In International Series in Operations Research and Management Science (Vol. 267, Issue April). https://doi.org/10.1007/978-3-319-71691-6_6

Restrepo, L., \& Gonzáles, J. (2007). From Pearson to Spearman. Revista Colombiana de Ciencias Pecuarias, 20(2), 183-192.

http://www.scielo.org.co/scielo.php?script=sci_arttext\&pid=S0120-06902007000200010

Ríos Ángeles, N. (2019). Tecnología aplicada a la reducción de costos de la logística de entrada en empresas importadoras de la industria alimentaría del Perú.

Rositas, J. (2004). La gestión bajo una cultura de calidad en México: una verificación empírica. (Quality management in Mexico: an empirical approach). Innovaciones de Negocios, 1(2), 265-276. http://www.web.facpya.uanl.mx/rev_in/Revistas/1.2/A6.pdf

Rositas, J., Badii, M. H., Castillo, J., \& Foroughbakhch, R. (2007). Técnicas de investigación basadas en sistemas de modelación estructurada. In Universidad Autónoma de Nuevo León \& M.H Badii \& J. Castillo (Eds.), Técnicas Cuantitativas en la Investigación (Primera ed).

Rositas Martínez, J. (2021). ALFA DE CRONBACH: Comprensión conceptual, verificación detallada de su cálculo y análisis crítico de su valor. Apuntes / Memoria en extenso.

Ruiz, M. A., Pardo, A., \& San Martín, R. (2010). Modelos de Ecuaciones Estructurales. Pepeles Del Psicólogo, 31(1), 34-45.

Vega Vilca, J. C., \& Guzman, J. (2011). Regresion PLS y PCA Como Solución al Problema de 
Multicolinealidad en Regresion Multiple. Revista de Matemática: Teoría y Aplicaciones, 18(1), 9. https://doi.org/10.15517/rmta.v18i1.2111 